\title{
Effects of Exercise Training with Proprioceptive Equipment on Proprioceptive and Functional Status of Children with Obstetrical Brachial Plexus Injury
}

\author{
Deran Oskay ${ }^{1}$, Edibe Ünal ${ }^{2}$, Gürsel Leblebicioğlu ${ }^{3}$, Zeynep Tuna ${ }^{1}$
}

\begin{abstract}
${ }^{1}$ Department of Physiotherapy and Rehabilitation, Gazi University, Faculty of Health Sciences, Ankara, Türkiye ${ }^{2}$ Department of Physiotherapy and Rehabilitation, Hacettepe University, Faculty of Health Sciences, Ankara, Türkiye

${ }^{3}$ Department of Orthopaedics and Traumatology, Hacettepe University, Faculty of Medicine, Ankara, Türkiye
\end{abstract}

Correspondence: Zeynep Tuna, Gazi University Faculty of Health Sciences, Department of Physiotherapy and Rehabilitation, Ankara, Türkiye E-mail: zeyneptuna6@yahoo.com
Received: 11 May 2016, Accepted: 14 April 2017 DOI:10.5799/jcei.328708

\section{A B S T R A C T}

Objective: Decreased proprioception in Obstetric Brachial Plexus Injury (OBPI) reduces the quality of functional movement. Hence, the aim of our study was to evaluate the effects of exercise training with additional proprioceptive equipments on the functional status and proprioceptive sense in children with OBPI.

Methods: Forty children with OBPI were randomly divided into two groups. Passive, active and active-assisted range of motion and stretching exercises were included in home-based program (HP) group while additional exercises with proprioceptive equipments were also added in the proprioceptive training (PT) group. After 6 weeks, proprioceptive perception and functional level were compared to pre-treatment results.

Results: Proprioceptive perception at the beginning and at the end of the movement increased in both groups $(p<0.05)$. The Active Movement Scale (AMS) scores increased significantly only in some movements in both groups $(p<0.05)$. AMS scores of shoulder abduction and external rotation in PT group were better than HP group $(p<0.05)$.

Conclusion: Home exercise program and proprioceptive exercise training both improve proprioceptive sense and functional status in children with OBPI. The proprioceptive exercise training seems to be more beneficial in improving functional movements of shoulder.

Key words: Exercise training, obstetrical brachial plexus injuries, proprioception

\section{INTRODUCTION}

Obstetric Brachial Plexus Injury (OBPI) develops due to traction of the Brachial Plexus (BP) during birth with variable severity [1]. Recovery can occur within a few months or may continue until the pre-school period depending on the type and severity of the nerve injury. However, with the delay in nerve regeneration the recovery level decreases and hence, results in muscle imbalance [2]. Muscle imbalance resulting in instabilities may cause contractures and deformities over time [3].

Proprioception is the ability to sense the position of body parts relative to each other and hence it controls joint movements. Proprioceptive sensation is a highly complex process including various systems from peripheral mechanoreceptors to central nervous system and incorporates with vestibular and visual systems. OBPI, because of the motor and sensory involvements, may impair proprioceptive mechanisms [4]. As presented in our previous study, proprioceptive perception impairment reduces the functional capacity of the affected extremity [5].

Functional capacity of a body part improves with enhanced proprioceptive input. Therefore, proprioceptive training is thought to improve functional capacity by providing appropriate movement sense. However, despite the numerous studies on different surgical procedures affecting the functional outcomes, studies about the effects of proprioceptive training on functional status are very poor. Therefore, the aim of the study is to evaluate the effects of proprioceptive training on functional status in children with OBPI. 


\section{METHODS}

This study was performed with patients referred to Hacettepe University Department of Physiotherapy and Rehabilitation for physiotherapy from a surgeon (G.L.) in Orthopaedics and Traumatology Department. The patients and their parents were invited according to inclusion and exclusion criteria to participate in the study at their first visit. The study design was approved ethically by the ethics committee and the informed consents of the parents were obtained.

Forty children with injuries of upper and/or middle trunks participated in the study. The inclusion criteria were being 7-12 years, involvement of upper and/or middle trunks (C5, C6, C7) and being able to participate in both assessments and exercise program. The children that received any previous physiotherapy program were excluded. The children were divided into two groups; a home program (HP) group $(n=20)$ and proprioceptive training added to home program group $(\mathrm{PT})(\mathrm{n}=20)$.

Exercise program included passive, active-assisted and active range of motion (ROM) exercises of shoulder, elbow and wrist joints and stretching exercises for the shoulder internal rotators, elbow flexors, and forearm pronators. The exercises were taught by an experienced physiotherapist to the parents and/or caregivers and they were instructed to perform the exercises three times a day. All the range of motion exercises were repeated 15 times within the possible full range and the stretching exercises were repeated 10 times with 30 seconds hold in each session. Home exercise program was performed similarly in both groups at home for 6 weeks while proprioceptive training was added in the PT group. The proprioceptive exercises are listed below however, minor modifications were possible according to the patient's tolerance. Proprioceptive exercises were instructed strictly to be performed in front of a mirror.

- Shoulder flexion, abduction, internal and external rotation with weights in a station workout form

- Shoulder flexion, abduction, internal and external rotation with the appropriate resistive band

- Bilateral shoulder elevation with the help of a stick

- Bilateral shoulder flexion and abduction with the help of a stick

- Shoulder elevation with an exercise ball in front of the mirror

- Throwing a ball while shoulder abduction, internal and external rotation are corrected manually by the supervisor

- Weight transferring bilaterally on the hands in the sitting position on an exercise ball

- Weight transferring bilaterally on the hands on a proprioception ball at various angles of shoulder flexion and abduction on the wall

\section{Assessment Tools}

The functional status and range of motion in the involved upper extremity joints were evaluated using the Active Movement Scale (AMS). AMS evaluates range of joint motion and is used instead of muscle strength tests that are not suitable for children [6].

Proprioceptive assessments were performed by Prosport 1000 PMS (Tumer Engineering Collective Company, Ankara). The device tests the sense of passive movement and passive repositioning in the shoulder and knee joints. The device was shown to be valid and reliable in the healthy adults in a previous study [7]. Proprioceptive testing was performed according to the protocols that had been used in our previous study [5]. The passive angular movement speed was determined as $2 \%$ [ [8]. The target angle of measurement was determined as $10-30-90 \%$ of the shoulder passive abduction angle $[4,8,9]$. The target angle in proprioception testing was determined as $10 \%, 30 \%$ and $90 \%$ of passive shoulder abduction for each patient.

Proprioceptive results were taken as a '-' value when the patient gets close to the target angle, and a ' + ' value when the patient passes the target angle. It was not important for values to be '-' or ' + ' as the absolute values were used in the analysis. A decrease in absolute values and getting close to ' 0 ' showed approximating to the target angle. All assessments were performed at the beginning and at the end of the 6-week exercise program.

\section{Statistical Analysis}

Statistical evaluations were performed with the SPSS 15.0 for Windows program. Mean plus minus standard deviation values were provided as descriptive statistics for data with normal distribution and "median (minimum-maximum) values for data without normal distribution. The Mann-Whitney U test was used for between-group comparisons and the Wilcoxon test was used to compare intra-group values before and after the treatment. Fisher's Exact test was used for between-group comparisons of gender distribution. Mean \pm standard deviation values were provided as additional information in addition to median (minimummaximum) values for the Mann-Whitney $U$ and Wilcoxon tests. Statistical significance was considered as $\mathrm{p}<0.05$.

\section{RESULTS}

The female/male ratio of patients according to randomization method was $9(45 \%) / 11(55 \%)$ and $8(40 \%) / 12(60 \%)$ in the HP and PT groups, respectively. Gender distribution of two groups was similar ( $p>0.05)$. The mean age was $9.50 \pm 1.87$ for the HP and $9.10 \pm 1.80$ for the PT group and were also similar in two groups $(\mathrm{p}=0.323)$.

Comparison of pre- and post-treatment results within groups showed a decrease in target angle of 10\% in PT group and 10\% and $90 \%$ in HP group $(<0.05)$ (Table 1). Comparison between groups showed no difference in terms of pre- and post-treatment proprioceptive changes $(\mathrm{p}>0.05)$ (Table 2$)$.

AMS scores of the joints were found to increase after treatment compared to pre-treatment scores in both groups. This increase was found to be significant in shoulder flexion, abduction, external 
Oskay D, et al. Exercise effects in OBPI

rotation and elbow flexion in HP group (Figure 1) and shoulder flexion, abduction, internal rotation, external rotation and elbow flexion in PT $(\mathrm{p}<0.05)$ (Figure 2). When AMS scores of two groups were compared, shoulder abduction and flexion scores were better in PT group $(\mathrm{p}<0,05)$ (Table 3$)$.

Table 1. Comparison of pre-treatment and post-treatment proprioceptive test results within groups.

\begin{tabular}{lcccc}
\hline Groups & $\begin{array}{c}\text { Target } \\
\text { Angle }\end{array}$ & $\begin{array}{c}\text { Pretreatment } \\
\text { values }\end{array}$ & $\begin{array}{c}\text { Posttreatment } \\
\text { values }\end{array}$ & $\mathbf{P}$ \\
\hline PT & $\% 10$ & 1.6 & 1.25 & $\mathbf{0 . 0 3 5}$ \\
& $\% 30$ & 4.8 & 3.3 & 0.155 \\
& $\% 90$ & 12.3 & 9.8 & 0.152 \\
\hline \multirow{2}{*}{ HP } & $\% 10$ & 1.25 & 0.9 & $\mathbf{0 . 0 0 8}$ \\
& $\% 30$ & 3.7 & 2.3 & 0.074 \\
& $\% 90$ & 8.45 & 5.7 & $\mathbf{0 . 0 0 8}$ \\
\hline
\end{tabular}

HP: Home program; PT: Proprioceptive training
Table 2. Comparison of pre-treatment and post-trea tment proprioceptive test results between groups

\begin{tabular}{lcccc}
\hline & $\begin{array}{c}\text { Target } \\
\text { Angle }\end{array}$ & $\begin{array}{c}\text { HP }(\mathbf{n}=20) \\
\mathbf{X} \pm \text { SS }\end{array}$ & $\begin{array}{c}\text { PT }(\mathbf{n}=20) \\
\mathbf{X} \pm \text { SS }\end{array}$ & $\mathbf{p}^{*}$ \\
\hline Pre-treatment & $\% 10$ & $1.6 \pm 1.14$ & $1.25 \pm 1.11$ & 0.33 \\
& $\% 30$ & $4.8 \pm 4.74$ & $3.7 \pm 3.84$ & 0.37 \\
& $\% 90$ & $12.3 \pm 13.4$ & $8.45 \pm 5.93$ & 0.88 \\
\hline Post-treatment & $\% 10$ & $1.25 \pm 1.06$ & $0.9 \pm 1.07$ & 0.24 \\
& $\% 30$ & $3.3 \pm 3.4$ & $2.3 \pm 2.4$ & 0.28 \\
& $\% 90$ & $9.8 \pm 10.4$ & $5.7 \pm 5.79$ & 0.13
\end{tabular}

HP: Home program; PT: Proprioceptive training, "Mann Whitney-U Test

\section{DISCUSSION}

This study showed that regular exercise program increased the proprioceptive perception and range of motion in the affected upper extremity. However, although additional proprioceptive training had no significant effect on the proprioception, it improved shoulder abduction and flexion ranges of movement more than the standard exercise group.

Table 3. Comparison of pre-treatment and post-treatment Active Movement Scale (AMS) scores between groups

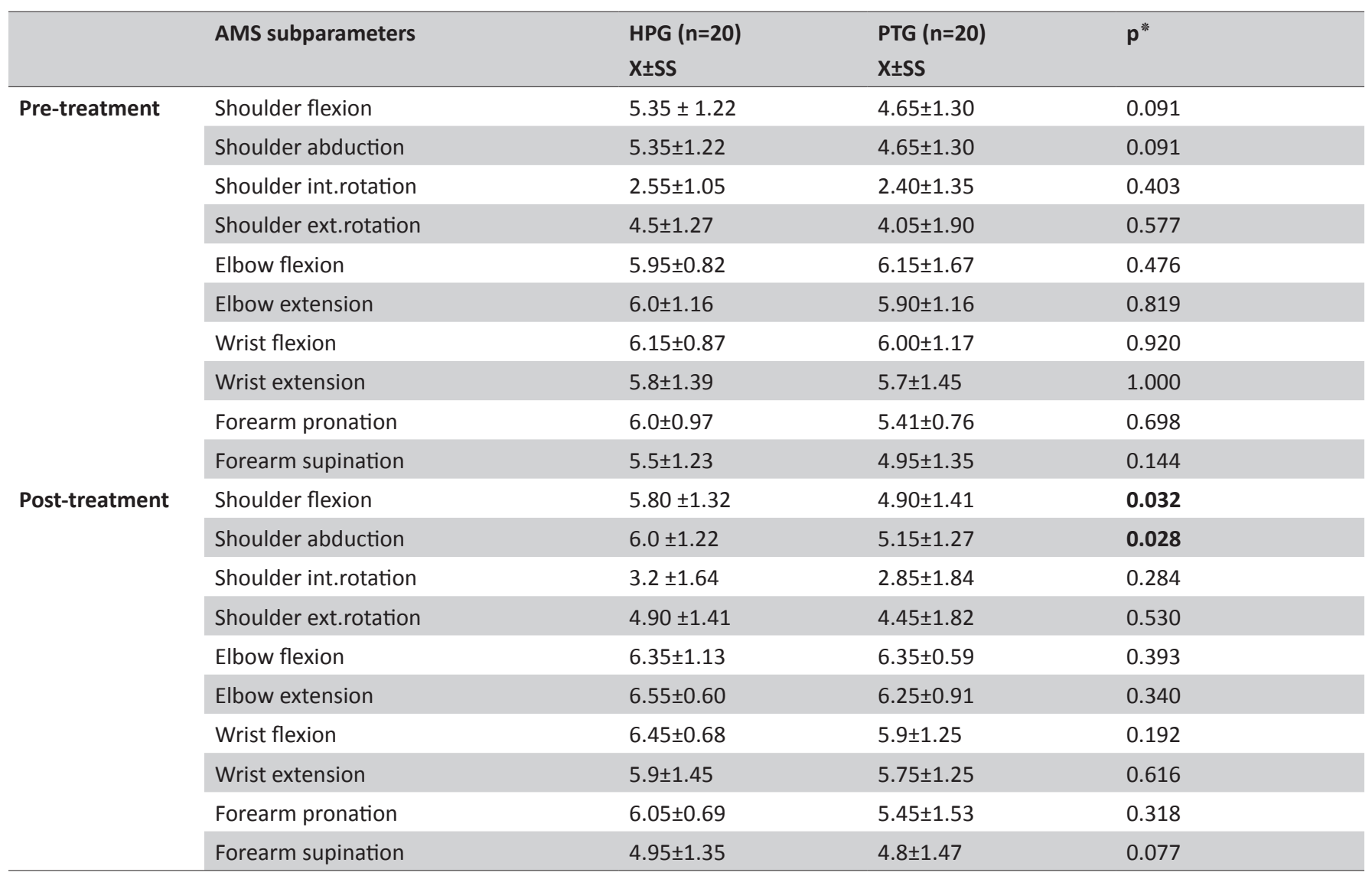

*Mann Whitney-U Test 
Physiotherapy interventions composed of stretching and ROM exercises have been shown to be essential from the early days of OBPI [10-12]. However, studies assessing the efficacy of physiotherapy in children with OBPI in primary school ages are extremely rare. Therefore, our study is one of the leading studies in the literature regarding the effects of exercise on proprioception in OBPI.

It is well known that the muscle spindle and golgi tendon organs are sensitive to tension, the Pacinian corpuscles to vibration, the Ruffini receptors to the static position of the joint and intraarticular pressure, and the free nerve endings within the joint to any chemical stimuli [13-16]. Any mechanical stimulus on the joint will lead to stimulation of these receptors. It is clear that the receptors are regularly stimulated and proprioceptive perception increased with proprioceptive exercises as well stretching and range of motion exercises. Therefore in our study, it may be concluded that proprioceptive benefits of different exercises like ROM and stretching exercises are similar.

In the literature, studies showed significant increases in proprioceptive perception with 6-week-exercise program in healthy individuals and 6-month exercise program in patients with joint injuries [17-19]. In our study, although 6-week exercise program increased the proprioceptive perception, this result was not statistically significant for every target angle. We, therefore, may conclude that longer periods are required in order to improve proprioception at all movement angles in impaired extremities such as those in OBPI.

We found the increase in proprioceptive perception to be statistically significant only at the beginning and end of the ROM. This may indicate that the recovery of perception of motion initiation may be seen earlier than for the following stages of movement. The improvement in the proprioceptive perception at the end of the range of motion can be explained by increased receptor sensitivity. This is the result of the extreme tension at the whole capsule and surrounding structures with extreme shoulder abduction due to stretching exercises at the final degrees of the shoulder range of motion [20].

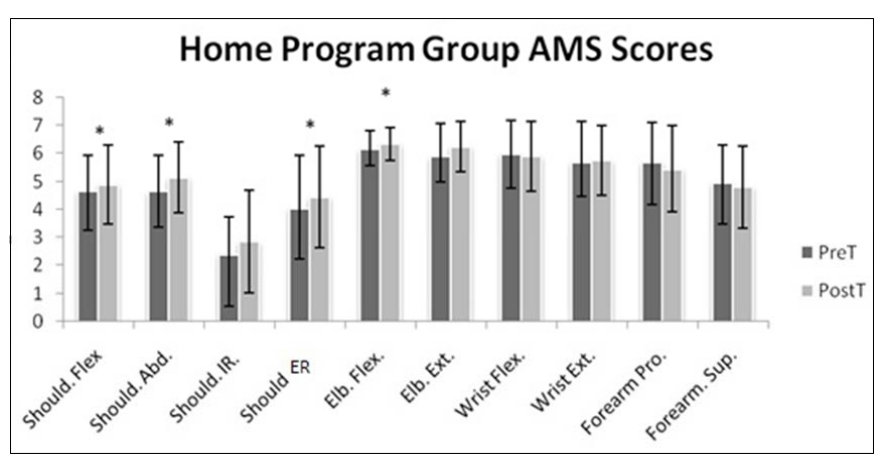

Figure 1. Active Movement Scale (AMS) scores of home program (HP) group (PreT: before treatment; PostT: after treatment), ${ }^{*} \mathrm{p}<$ 0.05
The most common deformities observed in all types of OBPI are shoulder internal rotation and adduction and elbow flexion contractures $[3,21]$. Hence, it is considered as the reason for the significant increase in the Active Movement Scale sub-parameters in the HP group only in shoulder external rotation and abduction and elbow extension in our study. We include stretching exercises in our study for shoulder adduction and internal rotation and elbow flexion contractures, which were quite possibly present in the patients.

Shoulder elevation lack is one of the greatest functional motor loss following OBPI [22-24]. The proprioceptive exercises used in our study were mainly designed to facilitate daily activities related to shoulder elevation. Therefore, shoulder abduction and flexion improved more in PT group in addition to increase in proprioceptive perception in both groups.

Proprioceptive sense plays an important role in producing coordinated movement with the antagonist and agonist muscle balance [25], regulation of true timing in multi-joint movements in the extremity [26], determination of movement direction and creation of skilled movement.

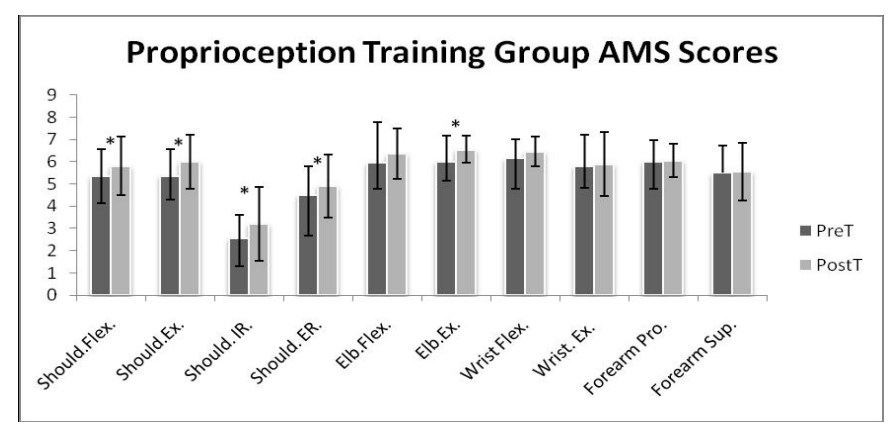

Figure 2. Active Movement Scale (AMS) scores of proprioception training (PT) group (PreT: before treatment; PostT: after treatment), ${ }^{*} p<0.05$

Therefore, improving the proprioceptive sensation has been subjected in some studies $[27,28]$. Those studies showed that proprioceptive sensations increase rapidly between the ages of 5 and 7 and it becomes stable after 7-12 years of age. The age range in our study was determined as 7-12 years by referring to these studies. We suggest that our age range did not affect the results of our study as there was no significant difference between the groups in terms of age and gender.

Proprioceptive training programs are mostly organized for 6 weeks for healthy subjects and for up to 6 months for the joints with various injuries. OBPI also affects intact muscles and the joints that are surrounded with the affected muscles since birth. Our primary finding was the increase in proprioceptive perception in both groups as a result of the 6-week exercise program but this result was not statistically significant for every target angle. Short follow-up period and the low number of patients are considered to be the main limitations of our study. Therefore, 
significant differences in more parameters may be achieved with longer treatment periods in larger sample sizes.

Proprioceptive perception should be strongly emphasized in children with OBPI as they have functional and physical impairments. Especially, physiotherapists working in the field should keep the proprioceptive assessment and treatment in mind as the functional status may be positively affected with the exercise programs.

Conflict of Interest: The authors report that they have no conflicts of interest.

Financial Disclosure: No financial support was received

Ethical approval: All procedures performed in studies involving human participants were in accordance with the ethical standards of the institutional and/or national research committee and with the 1964 Helsinki declaration and its later amendments or comparable ethical standards.

\section{REFERENCES}

1. Jennet RJ, Tarby TJ, Kraus RL. Erb's palsy constracted with Klumpke's and total palsy:different mechanism are involved. Am J Obstet Gynecol. 2002;186:1215-20

2. Gilbert A. Long term evaluation of brachial plexus surgery in obstetrical palsy. Hand Clins. 1995;11:583-94.

3. Kirkos JM, Papadopoulos IA. Late treatment of brachial plexus palsy secondary to birth injuries: rotational osteotomy of the proximal part of the humerus. J Bone Joint Surg. 1998; 80A:1477-83.

4. Magarey ME, Jones MA. Dynamic evaluation and early management of altered motor control around the shoulder complex. Man Ther. 2003;8:195-206.

5. Oskay D, Ünal E, Çetinkaya Ş. Do obstetrical brachial plexus injuries affect proprioceptive sense? Neurosciences. 2010;15:268-71.

6. Clarke HM, Curtis CG. Examination and Prognosis. In Gilbert A, ed. Brachial Plexus Injuries. London:Martin Dunitz. 2001:59-172.

7. Ulkar B, Kunduracioğlu B, Çetin C, et al. Effect of positioning and bracing on passive position sense of shoulder. Br J Sports Med. 2004;38:549-52.

8. Cuomo F, Birdzell MG, Zuckerman JD. The effect of degenerative arthritis and prosthetic arthroplasty on shoulder proprioception. J Shoulder Elbow Surg. 2005;14:345-48.

9. Birch R, Bonney G, Wynn Parry CB. Birth lesions of the brachial plexus. In Livingstone C, ed. Surgical Disorders of the Peripheral Nerves (209233) Edinburgh: Churchill Livingstone, 1998:209-33.

10. Eng $\mathrm{G}$, Binder $\mathrm{H}$, Getson P, et al. OBPP outcome with conservative management. Muscle Nerve. 1996;16:884-91.
11. Benjamin K. Part 2. Distinguishing physical characteristics and management of brachial plexus injuries. Adv Neonatal Care. 2005;5:24051.

12. Muhlig RS, Blaauw G, Sloof ACJ, et al. Conservative treatment of obstetrical brachial plexus palsy and rehabilitation. In Gilbert A, ed. Brachial Plexus Injuries. London:Martin Dunitz, 2001:173-187.

13. Grigg P. Peripheral neural mechanism in proprioception. J Sport Rehabil. 1994;3:2-17.

14. Cordo P, Carlton L, Bevan L, et al. Proprioceptive coordination of movement sequence: Role of velocity and position information. $\mathrm{J}$ Neurophysiol. 1994;71:1848-61

15. Johansson H, Sjölender P. Neurophysiology of joints. In Wright VEL, ed. Mechanics of Human Joint Physiology, Pathopyhsiology and Treatment. New York, 1993:243-90.

16. Grubb BD, Birrellm GJ, McQueen DS, Iggo A. The role of PGE in the sensitization of mechanoreceptors in normal and inflamated ankle joints of the rat. Exp Brain Res. 1991;184:383-92.

17. Swanik KA, Lephart SM, Swanik B, et al. The effect of shoulder plyometric training on proprioception and selected muscle performance characteristics. J Shoulder Elbow Surg. 2002;11:579-86.

18. Rogol IM, Ernst G, Perrin D. Open and closed kinetic chain exercises improve shoulder joint reposition sense equally in healthy subjects. $\mathrm{J}$ Athletic Train. 1998;33:315-18

19. Hilberg T, Herbsleb M, Puta C, et al. Physical training increase muscular strength and proprioceptive performance in haemophilic subjects. Haemophilia. 2003;9:86-93.

20. Jerosch JG. Effects of shoulder instability on joint proprioception. In Lephart SM, Fu FH, ed. Proprioception and neuromuscular control on joint instability. Pittsburg, 2002:247-64.

21. Waters P, Bae D. Effect of tendon transfers and extra articular soft tissue balancing on glenohumeral development in brachial plexus birth palsy. $\mathrm{J}$ Bone Joint Surg. 2005;87:320-25.

22. Benjamin K. Part 1. Injuries to the brachial plexus: mechanism of injury and identification of risk factors. Adv Neonatal Care. 2005;5:181-89.

23. Sunmire HF, DeMott RK. Erb's palsy without shoulder dystocia. Int J Gynaecol Obstet. 2000;78:253-56.

24. Shenaq SM, Berzin E, Lee R, et al. Brachial plexus birth injuries and current management. Clin Plast Surg. 1998;25:527-36.

25. Sainburg RL, Poizner H, Ghez C. Loss of proprioception produces deficits in interjoint coordination. J Neurophysiol. 1993;70:2136-47.

26. Cordo P, Carlton L, Bevan L, et al. Proprioceptive coordination of movement sequence: Role of velocity and position information. $\mathrm{J}$ Neurophysiol. 1994;71:1848-61.

27. Bairstow PJ, Lazslo JI. Kinestetic sensitivity to passive movements and its relation to motor development and motor control. Dev Med Child Neurol. 1981;23:606-16.

28. Hay L, Bard C, Ferrel C. Role of proprioceptive information in movement programing and control in 5 to 11-year-old children. Hum Mov Sci. 2005;24:139-54. 